\title{
Comment on "Minimization of maximum failure criterion of laminated composite shell structure by optimizing distributed-material orientation" by Shimoda M., Muramatsu Y., Tsukihara R.
}

\author{
V. Kobelev ${ }^{1}$
}

Received: 8 October 2019 / Revised: 12 December 2019 / Accepted: 8 January 2020 / Published online: 8 February 2020

(C) The Author(s) 2020

\begin{abstract}
The new "modified Tsai-Hill failure criterion" introduced in Shimoda et al. (SAMO, 2020) does not possess a convex fracture envelope for all possible set of its coefficients. An extended version of the "modified Tsai-Hill failure criterion" is introduced. The extended version encompasses several known failure criterions for the anisotropic materials. The convexity of the fracture envelope for composite materials and metallic alloys is briefly discussed. Albeit the convexity of the failure envelope is not necessary from the physical viewpoint, the convexity is favorable for the application of optimization methods.
\end{abstract}

Keywords Direction · Axis · Convexity

\section{Modified Tsai-Hill criterion}

In the article of Shimoda et al. (2020), the "modified Tsai-Hill failure criterion" was introduced. The proposed criterion could be considered to be pure phenomenological according to definition (Wu 1974). Equation (18) (Shimoda et al. 2020) reads:

$$
\begin{gathered}
f=\left(\frac{\sigma_{11}}{X_{1}\left(\sigma_{11}\right)}\right)^{2}-\frac{\sigma_{11}}{X_{1}\left(\sigma_{11}\right) X_{2}\left(\sigma_{22}\right)}+\left(\frac{\sigma_{22}}{X_{2}\left(\sigma_{22}\right)}\right)^{2} \\
+\left(\frac{\sigma_{12}}{S_{12}}\right)^{2}, \\
X_{1}\left(\sigma_{11}\right)=\left\{\begin{array}{ll}
X_{1 P}, & \sigma_{11}>0 \\
X_{1 M}, & \sigma_{11}<0
\end{array}, .\right. \\
X_{2}\left(\sigma_{22}\right)= \begin{cases}X_{2 P}, & \sigma_{22}>0 \\
X_{2 M}, & \sigma_{22}<0\end{cases}
\end{gathered}
$$

Responsible Editor: Helder C. Rodrigues

V. Kobelev

kobelev@imr.mb.uni-siegen.de

1 University of Siegen, 57076 Siegen, Germany
The new criterion (1) essentially linked to the directions of the reinforcement elements of the composite. For example, the axis " 1 " is preferably a fiber direction and axis " 2 " is normal to fiber direction. In the state of plane stress, the criterion possesses five positive coefficients $X_{1 P}, X_{1 M}, X_{2 P}, X_{2 M}, S_{12}$ and distinguishes the compression and tension stresses parallel and normal to fiber direction. Because the criterion is defined in a special coordinate system, linked to the directions of fibers or plies, the criterion is not invariant to the definition of coordinate system.

The function (1) is a homogeneous function of the second order in terms of stresses.

Each vector that initiates in the origin of the coordinate system of stress space intersects (crosses) only once the failure envelope. Thus, the failure envelope is a star-like surface.

The cited article uses the following values for optimization:

$$
\begin{aligned}
X_{1 P} & =1500 \mathrm{MPa}, X_{1 M}=1005 \mathrm{MPa}, X_{2 P}=40 \mathrm{MPa}, X_{2 M} \\
& =246 \mathrm{MPa}, S_{12}=68 \mathrm{MPa} .
\end{aligned}
$$

The new criterion (1) can be considered a generalization of the Tresca maximum shear criterion for isotropic materials as well. The Tresca criterion expresses the difference between the most positive and most negative principal stresses. For plane stress conditions, when one of the principal stresses is zero, one must use a different formula when the two plane 
principal stresses have the same sign and when they have different signs. If the signs of two plane principal stresses match, the zero third principal stress is used in the criterion. Alternatively, when the signs of two plane principal stresses are different, the zero principal stress is not used in the equation.

The Tsai-Wu criterion (Tsai and Wu 1971) renders this problem by adding linear terms to criterion. However, the additional linear terms abolish the homogeneity of the failure criterion (Groenwold and Haftka 2006). The citing article studied the optimization of safety factors of composite laminates with non-homogeneous failure criterions of Tsai-Wu. The optimization results are proved to be dependent upon the magnitude of the acting loads. Consequently, it is advantageous to use a homogeneous criterion, but the criterions have to distinguish the signs of stresses. The proposed criterion (1) differentiates the sign of the stresses and is suitable for the optimization purposes.

The application of the "modified Tsai-Hill failure criterion" (1) for the analysis of composites is questionable. The reason is the following. The correct failure criterion must define a convex region, which corresponds to admissible region. The proposed "failure criterion" defines for all possible values of stresses a non-convex failure envelope.

\section{Extended version of the Tsai-Hill criterion}

To analyze this problem of the criterion (1), we introduce the extended version of the "modified Tsai-Hill failure criterion" with two additional dimensionless parameters $m>0, \quad 0 \leq \kappa \leq$ 2:

$$
\begin{aligned}
F(m, \kappa)= & \left(\frac{\sigma_{11}}{X_{1}\left(\sigma_{11}\right)}\right)^{2 m}-\kappa\left(\frac{\sigma_{11}}{X_{1}\left(\sigma_{11}\right) X_{22}\left(\sigma_{22}\right)}\right)^{m} \\
& +\left(\frac{\sigma_{22}}{X_{2}\left(\sigma_{22}\right)}\right)^{2 m}+\left(\frac{\sigma_{12}}{S_{12}}\right)^{2 m} .
\end{aligned}
$$

Both criterions (1) and (3) are homogeneous functions. The degree of homogeneity of the function (3) is $2 m$.

Figure 1 shows the isolines (isarithm of the value 1 ) of the function $F(m, 0)$ for different exponents $m=1, m=2$, and $m=$ 4. The isolines of the value 1 represent the failure envelope of the criterion (3) for the plane stress state. The criterion (1) is similar to the Jenkins maximal stress criterion (Jenkins 1920), but with the quarter-elliptical boundaries fracture envelope instead of straight boundaries of Jenkins. In the state of plane stress, the criterion possesses five coefficients and is able to distinguish the compression and tension stresses parallel and normal to fiber direction. With the increasing exponent $m$, the failure envelope turns out to be increasingly rectangular. In the limit case $m \rightarrow \infty$, the failure envelope of the criterion (3) transforms into the failure envelope of the Jenkins criterion (Jenkins 1920).

Figure 2 displays the isolines (isarithm of the value 1) of the function $F(m, 1 / 2)$ for different exponents $m=1, m=2$, and $m=4$.

The isolines (isarithm of the value 1) of the function $F(m$, 1) for different exponents $m=1, m=2$, and $m=4$ are portrayed in Fig. 3. The isoline of the criterion (1) corresponds the isoline of the function (3) with the exponent $m=1$.

For the limit case $\kappa=2$, the failure criterion factorizes and the region is unbounded:

$$
\begin{aligned}
F(m, 2)= & {\left[\left(\frac{\sigma_{11}}{X_{1}\left(\sigma_{11}\right)}\right)^{m}-\left(\frac{\sigma_{22}}{X_{2}\left(\sigma_{22}\right)}\right)^{m}\right] } \\
& {\left[\left(\frac{\sigma_{11}}{X_{1}\left(\sigma_{11}\right)}\right)^{m}+\left(\frac{\sigma_{22}}{X_{2}\left(\sigma_{22}\right)}\right)^{m}\right]+\left(\frac{\sigma_{12}}{S_{12}}\right)^{2 m} . }
\end{aligned}
$$

The corresponding failure envelopes are displayed in Fig. 4.

The failure envelopes of the criterion (3) with the positive values of $\kappa$ looks similarly to failure envelopes of the Tsai-Wu criterion. Thus, the criterion (1) or (3) could be used for the approximation of the experimental data. Due to the homogeneity of the function, the criterions (1) and (3) are advantageous for the optimization methods.

\section{Convexity of failure envelopes}

Notably, that the failure envelope (zero-valued contour of the failure criterion (1) is not convex. Consider the absence of the shear stress $\sigma_{12}=0$. The failure envelope is the curve with four different equations in each quadrant $I, I I, I I I, I V$ :

$$
\begin{aligned}
f_{I}= & \left(\frac{\sigma_{11}}{X_{1 P}}\right)^{2}-\frac{\sigma_{11} \sigma_{22}}{X_{1 P} X_{2 P}}+\left(\frac{\sigma_{22}}{X_{2 P}}\right)^{2}, \text { in quadrant } I \\
: & \sigma_{11}>0, \sigma_{22}>0, \\
f_{I I}= & \left(\frac{\sigma_{11}}{X_{1 M}}\right)^{2}-\frac{\sigma_{11} \sigma_{22}}{X_{1 M} X_{2 P}}+\left(\frac{\sigma_{22}}{X_{2 P}}\right)^{2} \text {, in quadrant } I I \\
: & \sigma_{11}<0, \sigma_{22}>0, \\
f_{I I I}= & \left(\frac{\sigma_{11}}{X_{1 M}}\right)^{2}-\frac{\sigma_{11} \sigma_{22}}{X_{1 M} X_{2 M}}+\left(\frac{\sigma_{22}}{X_{2 M}}\right)^{2}, \text { in quadrant } I I I \\
: & \sigma_{11}<0, \sigma_{22}<0, \\
f_{I V}= & \left(\frac{\sigma_{11}}{X_{1 P}}\right)^{2}-\frac{\sigma_{11} \sigma_{22}}{X_{1 P} X_{2 M}}+\left(\frac{\sigma_{22}}{X_{2 M}}\right)^{2}, \text { in quadrant } I V \\
: & \sigma_{11}>0, \sigma_{22}<0 .
\end{aligned}
$$


Fig. 1 Contour lines (isarithms of the value 1$)$ of the function: $F(m$, 0)

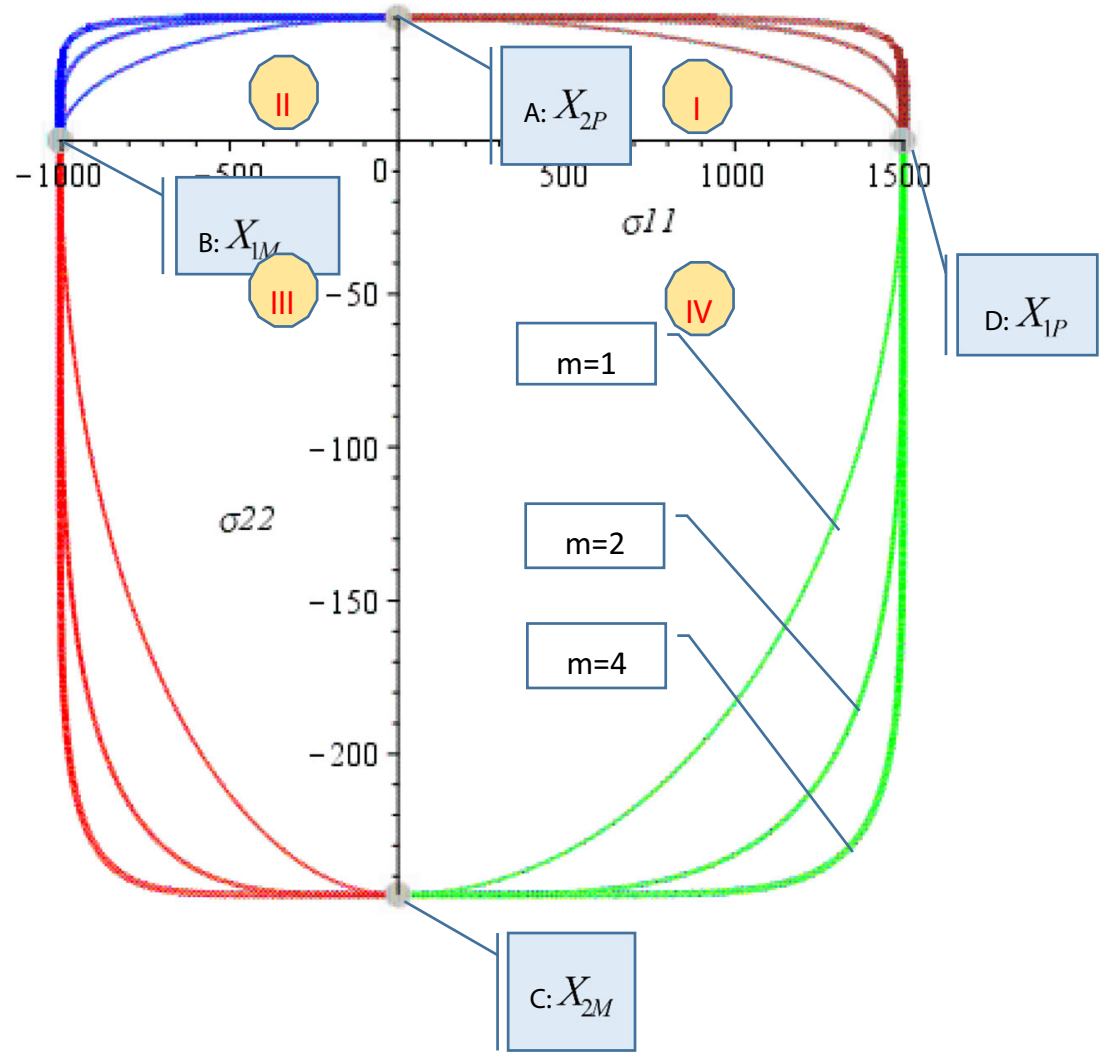

The curves are the quarters of ellipses in each quadrant and are all convex. The angles in the cross sections with the coordinate axes are sharp or obtuse. If the angle between the adjacent elliptical sections are sharp, the conical point occurs and the non-smooth curve is convex in the vicinity of the junction. Otherwise, if the angle is obtuse, such region in the
Fig. 2 Contour lines (isarithm of the value 1$)$ of the function: $F(m$, $1 / 2)$

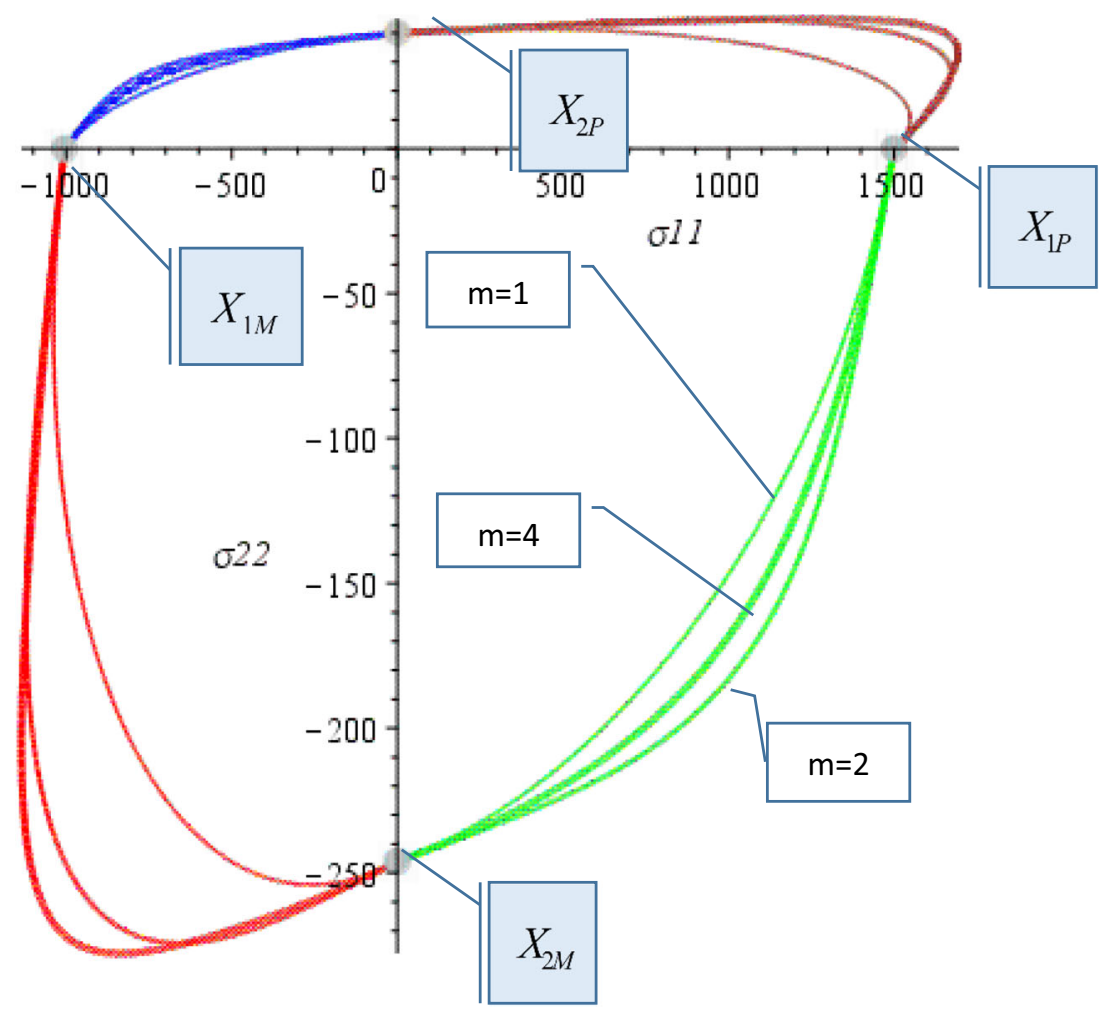


Fig. 3 Contour lines (isarithm of the value 1$)$ of the function: $F(m$, 1)

Fig. 4 Contour lines (isarithm of the value 1$)$ of the function: $F(m$, 2)
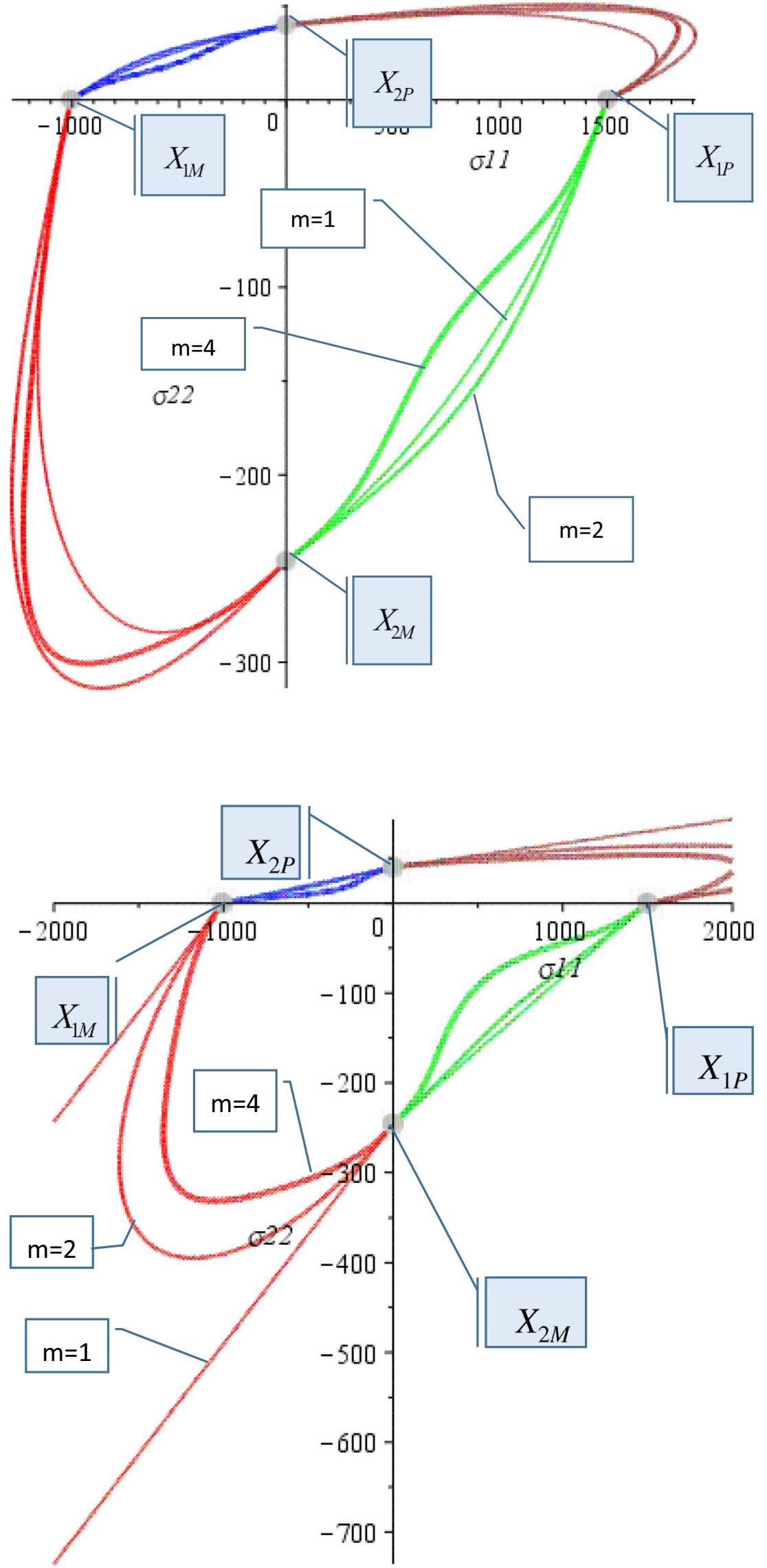
vicinity of junction point turns to be concave. The tangent vectors at both ends of each segment of the boundary curve use the curve equations for each section of (5) to (8).

The tangent vectors read:

$\boldsymbol{T}_{i}=\left[\begin{array}{l}\partial f_{i} / \partial \sigma_{11} \\ \partial f_{i} / \partial \sigma_{22}\end{array}\right], t_{i}=\frac{\boldsymbol{T}_{i}}{\left\|\boldsymbol{T}_{i}\right\|_{2}}$ for $i=A, B, C, D$

Using (9), the angles between tangent vectors are:

$\sin \alpha_{i}=\boldsymbol{t}_{i}^{+} \times \boldsymbol{t}_{i-1}^{-}$for $i=A, B, C, D$.

The calculation of the angles between the tangent vectors delivers the following values for the angles:

$$
\begin{aligned}
& \sin \alpha_{A}=-\frac{2 X_{2 P}\left(X_{1 M}-X_{1 P}\right)}{\sqrt{4 X_{1 P}^{2}+X_{2 P}^{2}} \sqrt{4 X_{1 M}^{2}+X_{2 P}^{2}}}, \\
& \sin \alpha_{B}=\frac{2 X_{1 M}\left(X_{2 M}-X_{2 P}\right)}{\sqrt{4 X_{2 P}^{2}+X_{1 M}^{2}} \sqrt{4 X_{2 M}^{2}+X_{1 M^{2}}}}, \\
& \sin \alpha_{C}=\frac{2 X_{2 M}\left(X_{1 M}-X_{1 P}\right)}{\sqrt{4 X_{1 M}^{2}+X_{2 M}^{2}} \sqrt{4 X_{1 P}^{2}+X_{2 M}^{2}}}, \\
& \sin \alpha_{D}=-\frac{2 X_{1 P}\left(X_{2 M}-X_{2 P}\right)}{\sqrt{4 X_{2 M^{2}+X_{1 P}^{2}} \sqrt{4 X_{2 P}^{2}+X_{1 P}^{2}}}} .
\end{aligned}
$$

Evidently, the signs of the angles $\alpha_{A}$ and $\alpha_{C}$ are opposite. Similarly, the signs of the angles $\alpha_{B}$ and $\alpha_{D}$ are also opposite. Consequently, the fracture envelope of the criterion (1) in two points is concave, if not all parameters in (2) are equal. The parameters are equal if and only if the material is isotropic in failure sense. For the isotropic material, the criterion (3) provides a generalization of the von Mises criterion.

\section{Convexity of failure envelopes for composite materials and structural metallic alloys}

The common failure criterions for fiber-reinforced composites possess the convex failure surfaces. Namely, almost all empirical and mechanism-based failure criterions for composites preserve the convexity of failure envelope (Cuntze 2006; Reifsnider and Masters 1987; Talreja and Varna 2016).

However, the considerably weaker requirement is obligatory in the field of composite mechanics. Explicitly, $\mathrm{Wu}$ (1974) requires that the failure envelope is star-convex from the origin stress space. Thus, the convexity of fracture envelopes is not essential for the anisotropic composites.

The analogous requirements appear in the different physical context. It has been long observed that plastic deformations induce anisotropy in initially isotropic metals and alloys (Meuwissen 1995). Since the work of Mises (1928), the convexity of the yield surface is commonly presumed for the anisotropic metals. Drucker (1964) showed that plastic solids in general must obey the normality rule. Drucker's method requires that the elastic range is star-convex from any point within the elastic range. In turn, this means that the yield surface must be convex (Ganczarski and Lenczowski 1997). The convexity of yield function in the stress space is associated with material stability. Crystallographic slip also guarantees convexity of polycrystal yield surfaces. The requirement of positive plastic dissipation does not necessarily exclude non-convex yield functions (Glüge and Bucci 2017). Their article has shown that the star convexity of the yield surface is sufficient to guarantee positivity of the work of stresses along the plastic strain increment.

Nevertheless, the convexity of failure envelope is favorable from the optimization viewpoint. Consider, for example, the optimization of strength and loading capacity of anisotropic elements (Kobelev 2019, § 4.2). In this problem, one searches the direction of the reinforcement (the fiber direction) that provides the minimum of the strength criterion, presuming the given stress tensor in the certain point. In the case of convex failure envelope, there is a unique orientation of the principal reinforcement. If the failure envelope is non-convex, there could be several orientations of the reinforcement, which deliver the minimum of strength criterion. Moreover, the optimal direction of reinforcement could undergo a finite jump from one to another local extrema even for an infinitesimally small variation of stress in the neighboring regions of the structural member.

\section{Conclusions}

The "modified Tsai-Hill failure criterion" (1) (Shimoda et al. 2020) and its extension (3) with two additional parameters are the phenomenological failure criterions, which are wellmatched for the optimization of composite structures. The criterions comprise as special cases several common failure criterions for anisotropic materials. The functions could be also interpreted as the yield surfaces for the flow theory of plasticity.

Notably, the failure envelopes of the criterions (1) and (3) are not convex. The convexity of the failure envelope of the anisotropic plastic materials is non-compulsory from the physical standpoint. However, the non-convexity of failure envelopes could lead to finite jumps of lamination angles in optimization problems. The convexity of the constraints is also advantageous for the numerical optimization algorithms (Bertsekas 2015).

Acknowledgements Open Access funding provided by Projekt DEAL.

\section{Compliance with ethical standards}

Conflict of interest The author declares that there is no conflict of interest. 
Replication of results The closed form formula for the failure criterion (3) is presented in the paper. This formula allows the instantaneous replication of the results. The evaluation of the parameters of equation (3) succeeds using the common methods of curve fitting for the experimental data.

Open Access This article is licensed under a Creative Commons Attribution 4.0 International License, which permits use, sharing, adaptation, distribution and reproduction in any medium or format, as long as you give appropriate credit to the original author(s) and the source, provide a link to the Creative Commons licence, and indicate if changes were made. The images or other third party material in this article are included in the article's Creative Commons licence, unless indicated otherwise in a credit line to the material. If material is not included in the article's Creative Commons licence and your intended use is not permitted by statutory regulation or exceeds the permitted use, you will need to obtain permission directly from the copyright holder. To view a copy of this licence, visit http://creativecommons.org/licenses/by/4.0/.

\section{References}

Bertsekas DP (2015) Convex optimization algorithms. Athena Scientific, Belmont

Cuntze RG (2006) Efficient 3D and 2D failure conditions for UD laminae and their application within the verification of the laminate design. Compos Sci Technol 66:1081-1096

Drucker D (1964) On the postulate of stability of material in the mechanics of continua. J Mec. 3:235-249

Ganczarski A, Lenczowski J (1997) On the convexity of the GoldenblatKopnov yield condition. Arch Mech 49(3):461-475
Glüge R, Bucci S (2017) Does convexity of yield surfaces in plasticity have a physical significance? Math Mech Solids:1-10. https://doi. org/10.1177/1081286517721599

Groenwold AA, Haftka RT (2006) Optimization with non-homogeneous failure criterions like Tsai-Wu for composite laminates. Struct Multidisc Optim 32:183-190. https://doi.org/10.1007/s00158-0060020-3

Jenkins CF (1920) Report on materials of construction used in aircraft and aircraft engines, Great Britain Aeronaut. Res. Committee

Kobelev V (2019) Design and analysis of composite structures for automotive applications. John Wiley \& Sons Ltd, Chichester

Meuwissen MHH (1995) Yield criterions for anisotropic elasto-plastic metals. (DCT rapporten; Vol. 1995.152) Eindhoven: Technische Universiteit Eindhoven

Mises RV (1928) Mechanik der plastischen Formänderung von Kristallen. Zeits f angew Math u Mech 8(3):161-185

Reifsnider KL, Masters JL (1987) Investigation of characteristic damage states in composite materials. ASME Paper No. 78-Aero-4, 3

Shimoda M, Muramatsu Y, Tsukihara R (2020) Minimization of maximum failure criterion of laminated composite shell structure by optimizing distributed-material orientation. Struct Multidisc Optim. https://doi.org/10.1007/s00158-019-02435-z

Talreja R, Varna J (eds) (2016) Modeling damage, fatigue and failure of composite materials, Woodhead Publishing Series in Composites, Science and Engineering: Number 65. Elsevier Ltd, Cambridge

Tsai SW, Wu EM (1971) A general theory of strength for anisotropic materials. J Compos Mater 5:58-80

Wu EM (1974) Phenomenological anisotropic failure criterions. In: Sendeckyj GP (ed) Mechanics of composite materials, vol 2. Academic Press, New York, pp 353-431

Publisher's note Springer Nature remains neutral with regard to jurisdictional claims in published maps and institutional affiliations. 